\title{
Confidence Intervals for Expected Abundance of Rare Species
}

\author{
David FLETCHER and Malcolm FADDY
}

\begin{abstract}
In many ecological research studies, abundance data are skewed and contain more zeros than might be expected. Often, the aim is to model abundance in terms of covariates, and to estimate expected abundance for a given set of covariate values. An approach that has been advocated recently involves the use of a conditional model. This allows one to separately model presence and abundance given presence, which should lead to a more complete understanding as to how the covariates influence abundance. The focus of this article is on the calculation of confidence intervals for expected abundance given particular values of the covariates. The standard Wald confidence interval is symmetric, and therefore unlikely to be of much use for skewed data, where reliable confidence intervals for abundance will generally be asymmetric. The purpose of this article is to show how to calculate a profile likelihood confidence interval for expected abundance using a conditional model.
\end{abstract}

Key Words: Conditional model; Negative binomial; Profile likelihood; Skewness; Zero-inflation.

\section{INTRODUCTION}

In many ecological research studies, abundance data often exhibit two features: a substantial proportion of the values are zero, and the remainder have a skewed distribution. In many types of study, the aim is to model the abundances in terms of one or more covariates, and to estimate the expected abundance for a given set of covariate values. We consider the use of a conditional-model approach for this purpose, as advocated by Welsh, Cunningham, Donnelly, and Lindenmayer (1996). This involves separately modeling (a) the presence of the species, and (b) the abundance of the species given that it is present (hereafter called the conditional abundance). This approach has the advantage that we can model these two aspects of the data separately, and thereby gain insight into whether they are being influenced by the covariates in different ways. Welsh et al. (1996) suggested using a truncated Poisson or truncated negative binomial distribution for the positive abundances. Dobbie and Welsh (2001) extended this type of model to deal with serial dependence in

David Fletcher is Associate Professor, Department of Mathematics and Statistics, University of Otago, P.O. Box 56, Dunedin, New Zealand (E-maildfletcher@maths.otago.ac.nz). Malcolm Faddy is Adjunct Professor, School of Mathematical Sciences, Queensland University of Technology, Brisbane, Queensland 4001, Australia.

(C) 2007 American Statistical Association and the International Biometric Society

Journal of Agricultural, Biological, and Environmental Statistics, Volume 12, Number 3, Pages 315-324

DOI: $10.1198 / 108571107 X 229322$ 
repeated measurements, while Barry and Welsh (2002) advocated the use of conditional generalized additive models. Martin et al. (2005) provided a useful review of the issue of zero-adjustment in ecological data.

Estimation of the expected abundance for a given set of covariate values is straightforward using a conditional model, and is based on the maximum likelihood estimates of the parameters from the two-component models. Welsh et al. (1996) suggested use of a Wald confidence interval around the estimate of the expected abundance. This interval is symmetric, being based on the assumption that the estimate has a sampling distribution that is approximately normal. The skewness we usually observe in abundance data suggests that an interval which contains the true expected abundance $95 \%$ of the time is likely to be asymmetric relative to the estimated expected abundance. Profile likelihood confidence intervals (Venzon and Moolgavkar 1988) are generally not symmetric and are therefore more informative than Wald intervals. The asymmetry will reflect differential uncertainty in the estimation, by having a different range of values on one side of the maximum likelihood estimate compared with the other. Such information is potentially useful, and is clearly not available from a symmetric confidence interval, such as the Wald interval. In addition, the Wald interval can be viewed as an approximation to the profile likelihood confidence interval (Brown, Cai, and DasGupta 2003; Cox and Hinkley 1974, p. 343; Lambert 1992). This was all recognized by Welsh et al. (1996), but they claimed that “. . . it is difficult to see how to apply this method [profile likelihood] to calculate confidence intervals for predicted values from the integrated model." The purpose of this article is to show how to calculate such intervals.

\section{MOTIVATING EXAMPLE}

The motivation for this article came from the first author's analysis of data from a study carried out by Eduardo Viloutta of the Department of Conservation, New Zealand. He was interested in assessing the relationship between abundance of a seaweed (Ecklonia radiata; hereafter Ecklonia) and that of a sea urchin (Evechinus chloroticus; hereafter Evechinus) in Fiordland, New Zealand. This particular species of sea urchin is found only in New Zealand and on subantarctic islands. The motivation for the study came from assessing the potential impact on the abundance of Ecklonia of establishing an Evechinus fishery. We therefore considered abundance of Ecklonia to be the response variable, with abundance of Evechinus being a predictor variable. The data we use to illustrate the method are part of a larger dataset, including a number of covariates that were also thought to influence the abundance of Ecklonia. We do not consider these here, for ease of presentation: the profile likelihood method can be applied quite generally, regardless of the number of predictor variables. Further information on the study and the complete dataset can be found in Fletcher, MacKenzie, and Villouta (2005). The data we use here are shown in Table 1.

At each of 103 locations, abundance of both Ecklonia and Evechinus was measured using a $25 \mathrm{~m}^{2}$ quadrat. There were 27 locations that showed 0 counts of Ecklonia, and Figure 1 shows a scatterplot of the abundances of the two species (the circled observation is discussed in Section 4). 
Table 1. Data on the relationship between abundance of Ecklonia radiata (plants/quadrat) and Evechinus chloroticus (individuals/quadrat), measured using a $25 \mathrm{~m}^{2}$ quadrat at each of 103 locations.

\begin{tabular}{|c|c|c|c|c|c|}
\hline Ecklonia & Evechinus & Ecklonia & Evechinus & Ecklonia & Evechinus \\
\hline 0 & 3 & 2 & 0 & 46 & 28 \\
\hline 0 & 29 & 3 & 14 & 48 & 4 \\
\hline 0 & 38 & 3 & 115 & 49 & 0 \\
\hline 0 & 0 & 6 & 22 & 56 & 29 \\
\hline 0 & 18 & 8 & 53 & 57 & 2 \\
\hline 0 & 6 & 8 & 15 & 57 & 15 \\
\hline 0 & 18 & 11 & 64 & 57 & 11 \\
\hline 0 & 11 & 12 & 28 & 58 & 0 \\
\hline 0 & 19 & 12 & 52 & 58 & 28 \\
\hline 0 & 32 & 15 & 14 & 59 & 0 \\
\hline 0 & 33 & 16 & 0 & 59 & 9 \\
\hline 0 & 133 & 19 & 11 & 61 & 0 \\
\hline 0 & 19 & 20 & 11 & 64 & 1 \\
\hline 0 & 0 & 20 & 20 & 67 & 0 \\
\hline 0 & 13 & 20 & 14 & 70 & 0 \\
\hline 0 & 5 & 22 & 7 & 72 & 0 \\
\hline 0 & 5 & 24 & 5 & 73 & 5 \\
\hline 0 & 27 & 25 & 0 & 79 & 2 \\
\hline 0 & 39 & 26 & 12 & 81 & 3 \\
\hline 0 & 110 & 28 & 20 & 81 & 8 \\
\hline 0 & 31 & 30 & 11 & 84 & 0 \\
\hline 0 & 50 & 30 & 11 & 87 & 0 \\
\hline 0 & 0 & 31 & 20 & 88 & 25 \\
\hline 0 & 34 & 33 & 13 & 89 & 2 \\
\hline 0 & 220 & 33 & 52 & 94 & 28 \\
\hline 0 & 78 & 33 & 19 & 96 & 2 \\
\hline 0 & 27 & 33 & 29 & 96 & 4 \\
\hline 1 & 23 & 34 & 24 & 103 & 29 \\
\hline 1 & 15 & 35 & 25 & 118 & 13 \\
\hline 1 & 15 & 38 & 0 & 137 & 0 \\
\hline 2 & 35 & 38 & 19 & 157 & 4 \\
\hline 2 & 34 & 39 & 0 & 168 & 5 \\
\hline 2 & 15 & 40 & 35 & 203 & 50 \\
\hline 2 & 19 & 44 & 0 & & \\
\hline 2 & 16 & 45 & 0 & & \\
\hline
\end{tabular}




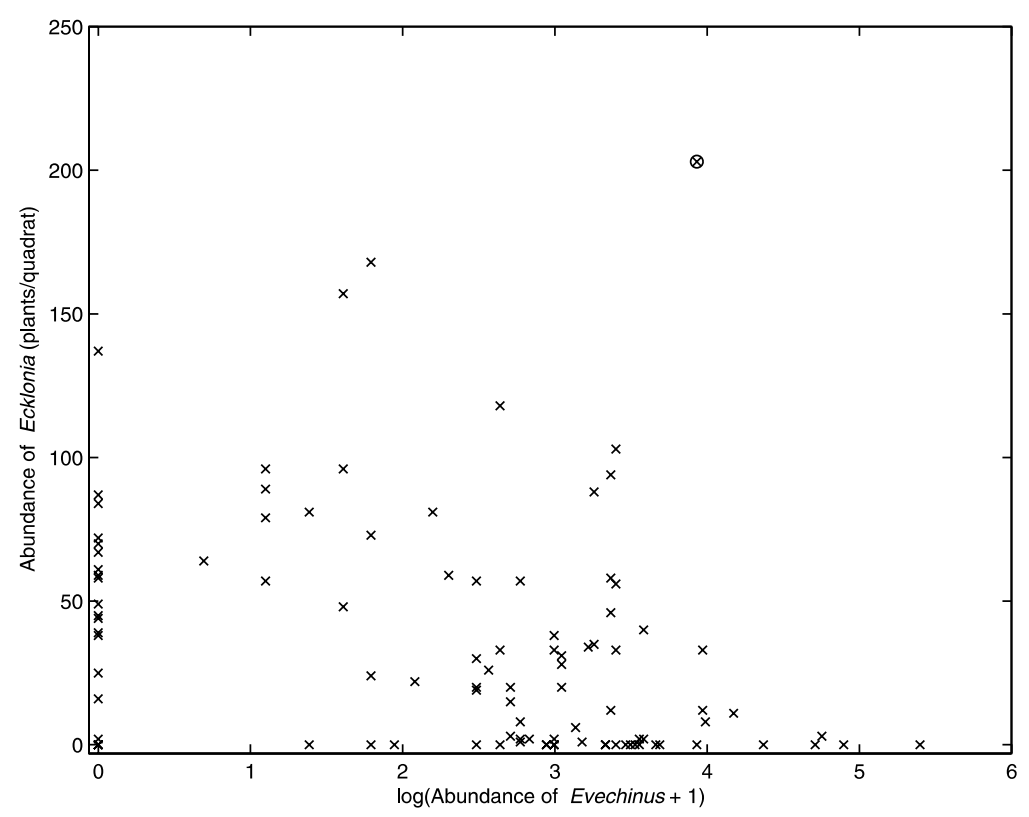

Figure 1. Scatterplot of the abundance of Ecklonia and the abundance of Evechinus.

The objective of the analysis is to estimate the expected abundance of Ecklonia (plants/ quadrat) for a given abundance of Evechinus (individuals/quadrat). Preliminary analyses suggested an approximately linear relationship between Ecklonia and $\log ($ Evechinus +1$)$, so we used the latter as our predictor variable in each of the two-component models.

\section{PROFILE LIKELIHOOD INTERVAL FOR THE CONDITIONAL MODEL}

Let $Y$ be the abundance of the species of interest (response variable) and let $Z$ indicate presence (1) or absence ( 0 ) of the species. We model $Z$ and $Y \mid Z=1$ in terms of covariates $\mathbf{x}=\left(x_{0}, x_{1}, \ldots, x_{p}\right)^{\mathrm{T}}$ and $\mathbf{w}=\left(w_{0}, w_{1}, \ldots, w_{q}\right)^{\mathrm{T}}$, respectively. Note that we will usually wish to include an intercept in each of these models, and so we have $x_{0}=w_{0}=1$. If we assume a truncated negative binomial distribution for $Y \mid Z=1$, we have

$$
\operatorname{Pr}(Y=0 \mid \mathbf{x})=\operatorname{Pr}(Z=0 \mid \mathbf{x})=1-\pi(\mathbf{x})
$$

and

$$
\begin{aligned}
\left.\operatorname{Pr}(Y=r \mid \mathbf{x}, \mathbf{w})=\pi(\mathbf{x}) \frac{\Gamma(r+}{\Gamma(r+1)}\right) & \Gamma(k) \\
\times & {\left[1-\left\{1+\frac{\lambda(\mathbf{w})}{k}\right\}^{-k}\right]^{-1}\left\{\frac{\lambda(\mathbf{w})}{k}\right\}^{r}\left\{1+\frac{\lambda(\mathbf{w})}{k}\right\}^{-(r+k)} }
\end{aligned}
$$


for $r=1,2,3, \ldots$, where $\pi(\mathbf{x})=\operatorname{Pr}(Z=1 \mid \mathbf{x})$ is the probability that the species is present, and $\lambda(\mathbf{w})$ is the mean of the untruncated negative binomial distribution, the mean of the truncated version being

$$
E(Y \mid Z=1)=\lambda(\mathbf{w})\left\{1-\left(1+\frac{\lambda(\mathbf{w})}{k}\right)^{-k}\right\}^{-1}
$$

(Johnson, Kemp, and Kotz 2005, chap. 5).

Using a logistic link and log link for $\pi(\mathbf{x})$ and $\lambda(\mathbf{w})$, respectively, we have

$$
\log \left\{\frac{\pi(\mathbf{x})}{1-\pi(\mathbf{x})}\right\}=\mathbf{x}^{\mathrm{T}} \boldsymbol{\beta},
$$

and

$$
\log \{\lambda(\mathbf{w})\}=\mathbf{w}^{\mathrm{T}} \boldsymbol{\theta},
$$

where $\boldsymbol{\beta}=\left(\beta_{0}, \beta_{1}, \ldots, \beta_{p}\right)^{\mathrm{T}}$ and $\boldsymbol{\theta}=\left(\theta_{0}, \theta_{1}, \ldots, \theta_{q}\right)^{\mathrm{T}}$ are vectors of unknown parameters.

Suppose we have abundances $y_{i}$, of which $m$ are positive $(i=1,2, \ldots, n)$. Let the corresponding values for the covariates be denoted $\mathbf{x}_{i}=\left(1 x_{1 i}, \ldots, x_{p i}\right)^{\mathrm{T}}$ and $\mathbf{w}_{i}=$ $\left(1 w_{1 i}, \ldots, w_{q i}\right)^{\mathrm{T}}(i=1,2, \ldots, n)$. The log-likelihood for the data is given by

$$
\begin{aligned}
l(\boldsymbol{\beta}, \boldsymbol{\theta}, k)=\sum_{y_{i}=0} \log \left(1-\pi_{i}\right)+\sum_{y_{i}>0} \log \pi_{i}+m\{k \log k-\log \Gamma(k)\} \\
+\sum_{y_{i}>0}\left[\log \Gamma\left(y_{i}+k\right)-\log \Gamma\left(y_{i}+1\right)+y_{i} \log \lambda_{i}\right. \\
\left.-\left(y_{i}+k\right) \log \left(k+\lambda_{i}\right)-\log \left\{1-\left(1+\frac{\lambda_{i}}{k}\right)^{-k}\right\}\right]
\end{aligned}
$$

where $k>0$, and we have

$$
\pi_{i}=\pi\left(\mathbf{x}_{i}\right)=\left\{1+\exp \left(-\mathbf{x}_{i}^{\mathrm{T}} \boldsymbol{\beta}\right)\right\}^{-1},
$$

and

$$
\lambda_{i}=\lambda\left(\mathbf{w}_{i}\right)=\exp \left(\mathbf{w}_{i}^{\mathrm{T}} \boldsymbol{\theta}\right) .
$$

Note that the "overdispersion" parameter $k$ is assumed to not depend on any of the covariates.

Suppose we wish to estimate the expected abundance for particular values of the covariates, $\mathbf{x}_{0}=\left(1 x_{10}, \ldots, x_{p 0}\right)^{\mathrm{T}}$ and $\mathbf{w}_{0}=\left(1 w_{10}, \ldots, w_{q 0}\right)^{\mathrm{T}}$. This is given by

$$
\begin{aligned}
\mu_{0} & =E\left(Y \mid \mathbf{x}_{0}, \mathbf{w}_{0}\right) \\
& =\pi_{0} \lambda_{0}\left\{1-\left(1+\frac{\lambda_{0}}{k}\right)^{-k}\right\}^{-1}
\end{aligned}
$$

where, for ease of notation, we use $\pi_{0}$ and $\lambda_{0}$ to denote $\pi\left(\mathbf{x}_{0}\right)$ and $\lambda\left(\mathbf{w}_{0}\right)$, respectively. The maximum likelihood estimate of $\mu_{0}$ is given by

$$
\hat{\mu}_{0}=\hat{\pi}_{0} \hat{\lambda}_{0}\left\{1-\left(1+\frac{\hat{\lambda}_{0}}{\hat{k}}\right)^{-\hat{k}}\right\}^{-1},
$$


where

and

$$
\hat{\pi}_{0}=\left\{1+\exp \left(-\mathbf{x}_{0}^{\mathrm{T}} \hat{\boldsymbol{\beta}}\right)\right\}^{-1}
$$

$$
\hat{\lambda}_{0}=\exp \left(\mathbf{w}_{0}^{\mathrm{T}} \hat{\boldsymbol{\theta}}\right)
$$

and $\hat{\boldsymbol{\beta}}, \hat{\boldsymbol{\theta}}$, and $\hat{k}$ are the maximum likelihood estimates of $\boldsymbol{\beta}, \boldsymbol{\theta}$, and $k$, respectively.

Calculation of a profile likelihood interval for some quantity of interest involves maximizing the log-likelihood under the constraint that that quantity takes a fixed value $v$, say. The profile log-likelihood is the maximized likelihood as a function of $v$. The quantity of interest here is the unconditional mean (3.2), and so the relevant constrained maximization is equivalent to unconstrained maximization of:

$$
l(\boldsymbol{\beta}, \boldsymbol{\theta}, k)-\gamma \pi_{0} \lambda_{0}\left\{1-\left(1+\frac{\lambda_{0}}{k}\right)^{-k}\right\}^{-1},
$$

where $\gamma$ is a Lagrange multiplier (Khuri 1993, p. 287). We maximize (3.3) for a range of values of $\gamma$, and the corresponding values of $\mu_{0}$ are given by substituting the resulting estimates of $\pi_{0}, \lambda_{0}$, and $k$ into (3.2). Thus, varying $\gamma$ amounts to varying $\mu_{0}$ with $\gamma=0$ corresponding to the maximum likelihood estimate $\hat{\mu}_{0}$.

Note that use of a Lagrange multiplier is equivalent to writing one of the original parameters in (3.1) in terms of $\mu_{0}$ and working with the resulting log-likelihood as a function of the remaining original parameters and $\mu_{0}$. This is true even when such a substitution cannot be performed explicitly. Note also that in performing the maximization of (3.3) numerically, it is a good idea to replace

$$
\pi_{0} \lambda_{0}\left\{1-\left(1+\frac{\lambda_{0}}{k}\right)^{-k}\right\}^{-1}
$$

by some bounded monotone function of it, such as $x /(1+x)$, to prevent this component becoming too large.

The profile log-likelihood for $\mu_{0}$ is defined as

$$
l_{p}\left(\mu_{0}\right)=l\{\tilde{\boldsymbol{\beta}}, \tilde{\boldsymbol{\theta}}, \tilde{k}\}
$$

where $\tilde{\boldsymbol{\beta}}, \tilde{\boldsymbol{\theta}}$, and $\tilde{\boldsymbol{k}}$ are the values of $\boldsymbol{\beta}, \boldsymbol{\theta}$, and $k$ that maximize (3.3) for a given value of $\mu_{0}$ (or equivalently $\gamma$ ). Note that the values of $\tilde{\boldsymbol{\beta}}, \tilde{\boldsymbol{\theta}}$, and $\tilde{k}$ will depend on $\mu_{0}$, and that we have suppressed this dependence for ease of notation. Large-sample likelihood theory (Venzon and Moolgavkar 1988) tells us that

$$
w\left(\mu_{0}\right)=2\left\{l_{p}\left(\hat{\mu}_{0}\right)-l_{p}\left(\mu_{0}\right)\right\}
$$

will have a distribution which is approximately $\chi_{1}^{2}$. This leads to a $95 \%$ profile likelihood confidence interval being defined as the two values of $\mu_{0}$ that satisfy $w\left(\mu_{0}\right)=3.8415$, with 3.8415 being the 95 th percentile of the $\chi_{1}^{2}$ distribution.

The estimates $\hat{\boldsymbol{\beta}}, \hat{\boldsymbol{\theta}}, \hat{k}, \tilde{\boldsymbol{\beta}}, \tilde{\boldsymbol{\theta}}$, and $\tilde{k}$ are all obtained using numerical methods. For the example in the next section, we performed the calculations in MATLAB, using the NelderMead simplex algorithm for numerical optimization. 
Table 2. Parameter estimates for the two components of the conditional model, together with $95 \%$ profile likelihood confidence intervals.

\begin{tabular}{lcrc}
\hline \hline \multicolumn{1}{c}{ Model } & Parameter & Estimate & \multicolumn{1}{c}{ CI } \\
\hline Probability of presence & $\beta_{0}$ & 2.18 & $(1.17,3.41)$ \\
log-likelihood $=-55.94$ & $\beta_{1}$ & -0.45 & $(-0.85,-0.10)$ \\
& & & \\
Conditional abundance & $\theta_{0}$ & 4.20 & $(3.78,4.65)$ \\
log-likelihood $=-366.71$ & $\theta_{1}$ & -0.18 & $(-0.36,-0.0077)$ \\
& $k$ & 1.01 & $(0.67,1.43)$ \\
\hline
\end{tabular}

\section{EXAMPLE}

As we focus on a single covariate in our example, we have $\mathbf{x}=\mathbf{w}, p=q=1$, and there are five parameters: $\beta_{0}, \beta_{1}, \theta_{0}, \theta_{1}$, and $k$. Table 2 shows the maximum likelihood estimates for these parameters, together with $95 \%$ profile likelihood confidence intervals.

Figure 2 shows the profile log-likelihood as a function of the expected abundance of Ecklonia when the abundance of Evechinus is zero. The "cut" for a $95 \%$ confidence interval is also shown, together with a quadratic approximation to this profile, corresponding to a Wald interval. The asymmetry about the maximum likelihood estimate of the profile log-

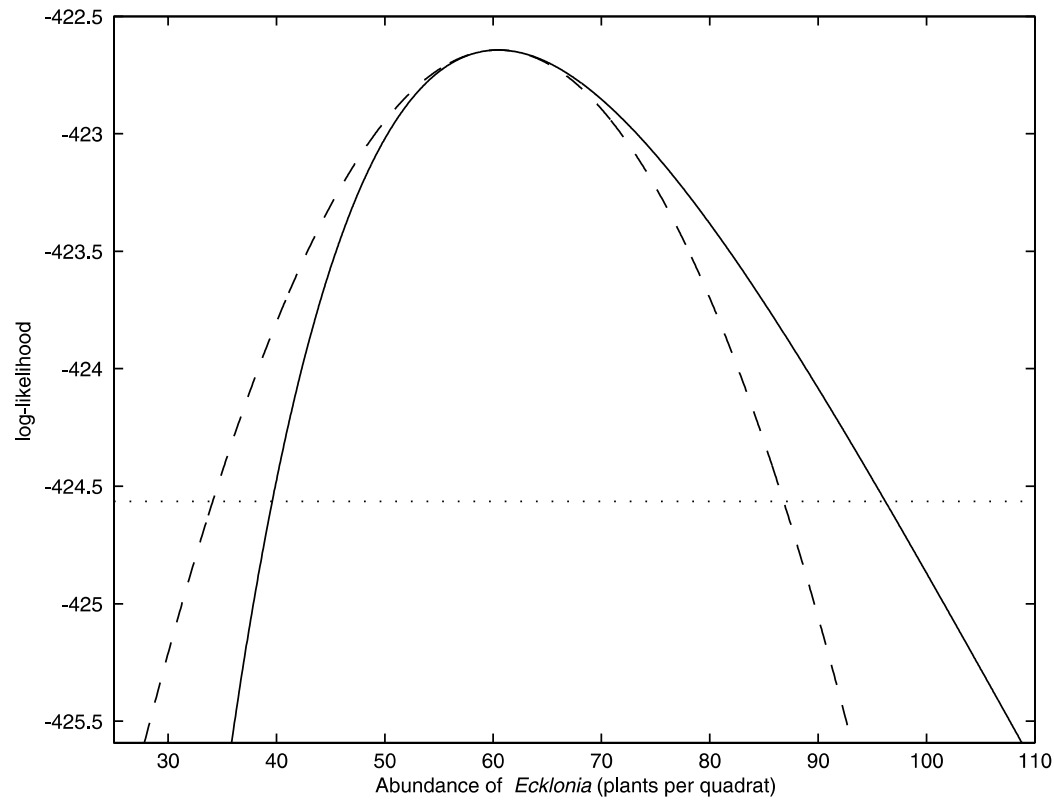

Figure 2. Profile log-likelihood for expected abundance of Ecklonia when abundance of Evechinus is zero (- - ), with a quadratic approximation $\left(--_{-}\right)$and the "cut" for a $95 \%$ confidence interval $(\cdots)$. 




Figure 3. Estimates and 95\% profile likelihood confidence intervals for the abundance of Ecklonia, over a range of values for the abundance of Evechinus.

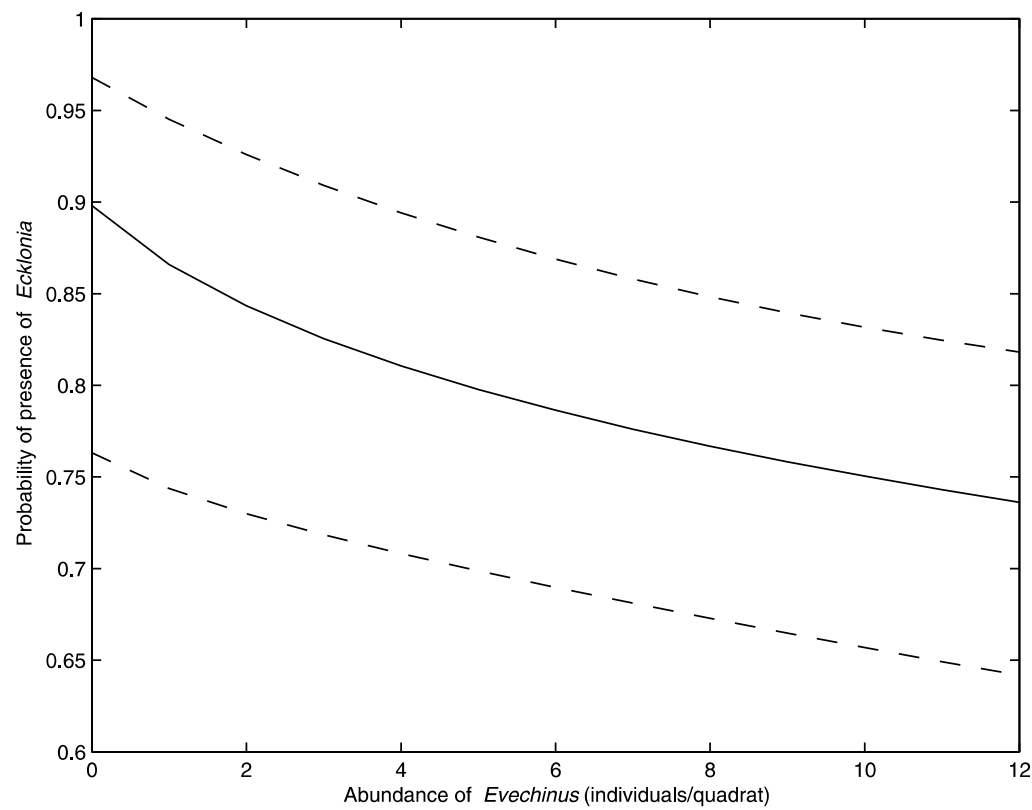

Figure 4. Estimates and 95\% profile likelihood confidence intervals for the probability of presence of Ecklonia, over a range of values for the abundance of Evechinus. 


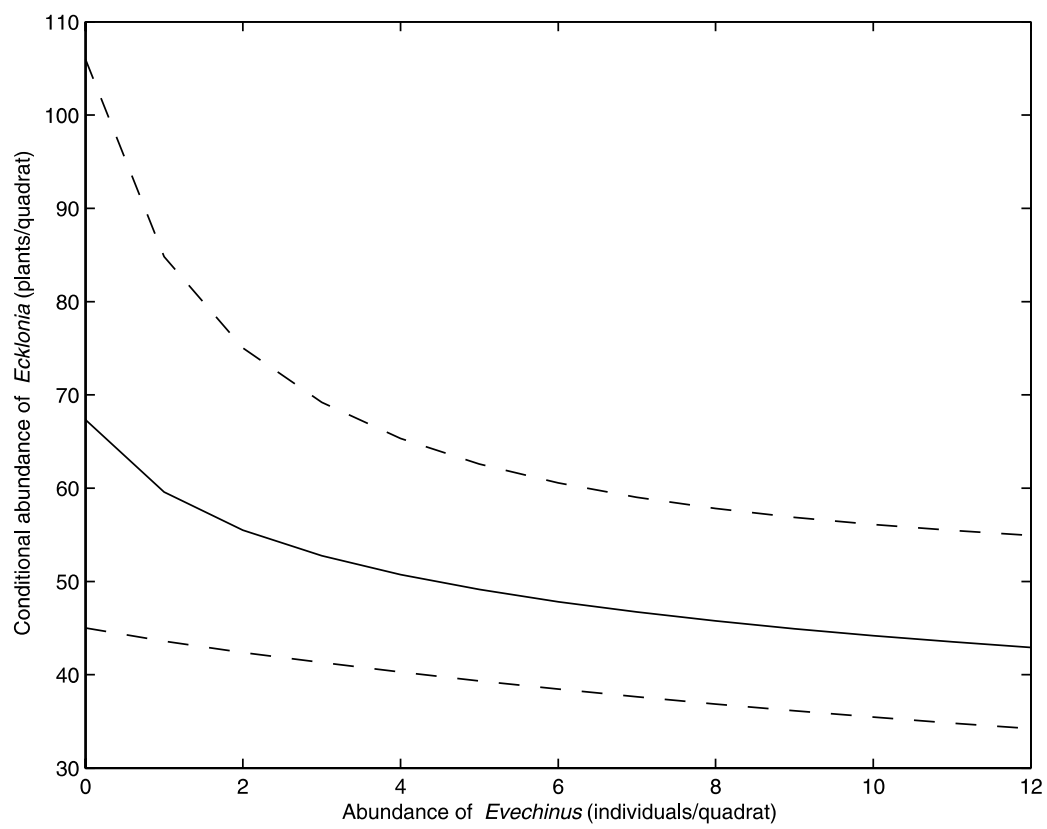

Figure 5. Estimates and 95\% profile likelihood confidence intervals for the conditional abundance of Ecklonia, over a range of values for the abundance of Evechinus.

likelihood interval shows that there is less uncertainty in the estimation below the maximum likelihood estimate than above it.

Figure 3 shows estimates and 95\% profile likelihood confidence intervals for the expected abundance of Ecklonia over a range of values for the abundance of Evechinus. The confidence intervals clearly aid interpretation of the effect of Evechinus abundance on Ecklonia. In addition, the asymmetry achieved using a profile likelihood interval is intuitively sensible.

The generalized Pearson statistic (sum of squared standardized residuals) is 99.3 on 98 df, suggesting an adequate fit. The circled observation in Figure 1 has the largest standardized residual, with a tail probability of 0.0014 . This might be considered an outlier, but it is not altogether extreme in a sample of 103 observations. As this observation also appears influential, we checked the effect of removing it from the analysis. The difference on the fitted model is not great (the log-likelihood increased by 0.76), with the estimates of $\theta_{0}, \theta_{1}$, and $k$ (Table 2 ) changing by $+0.11,-0.08$, and +0.12 , respectively (the estimates of $\beta_{0}$ and $\beta_{1}$ are unaffected). The next largest standardized residual corresponds to a tail probability of 0.026, which would seem unremarkable in a sample of 103 observations.

Although they are not the focus of this article, we also calculated estimates and $95 \%$ profile likelihood confidence intervals for the probability of presence of Ecklonia (Figure 4) and the conditional abundance of Ecklonia (Figure 5), respectively. These graphs complement Figure 2, in that they aid understanding as to how the two components of abundance of Ecklonia are influenced by abundance of Evechinus.

Finally, we note that the approach we present here is quite general and could be applied, 
in principle, to any situation where we wish to calculate a confidence interval for the expected value from a statistical model. It is likely to be of most value when a symmetric confidence interval might provide a poor reflection of the uncertainty in the estimate of the expected value, due to asymmetry of the profile log-likelihood function.

[Received July 2006. Revised February 2007.]

\section{REFERENCES}

Barry, S. C., and Welsh, A. H. (2002), "Generalized Additive Modelling and Zero Inflated Count Data," Ecological Modelling, 157, 179-188.

Brown, L. D., Cai, T., and DasGupta, A. (2003), "Interval Estimation in Exponential Families," Statistica Sinica, $13,19-49$.

Cox, D. R., and Hinkley, D. V. (1974), Theoretical Statistics, London: Chapman and Hall.

Dobbie, M. J., and Welsh, A. H. (2001), "Modelling Correlated Zero-Inflated Count Data," Australian and New Zealand Journal of Statistics, 43, 431-444.

Fletcher, D. J., MacKenzie, D. I., and Villouta, E. (2005), "Modelling Skewed Data with Many Zeros: A Simple Approach Combining Ordinary and Logistic Regression," Environmental and Ecological Statistics, 12, 45-54.

Johnson, N.L., Kemp, A.W., and Kotz, S. (2005), Univariate Discrete Distributions (3rd ed.), New York: Wiley.

Khuri, A.I. (1993), Advanced Calculus with Applications in Statistics, New York: Wiley.

Lambert, D. (1992), “Zero-Inflated Poisson Regression, With an Application to Defects in Manufacturing,” Technometrics, 34, 1-14.

Martin, T. G., Wintle, B. A., Rhodes, J. R., Kuhnert, P. M., Field, S. A., Low-Choy, S. J., Tyre, A. J., and Possingham, H. P. (2005), "Zero Tolerance Ecology: Improving Ecological Inference by Modelling the Source of Zero Observations," Ecology Letters, 8, 1235-1246.

Stephenson, G. (1961), Mathematical Methods for Science Students, London: Longmans.

Venzon, D. J., and Moolgavkar, S. H. (1988), “A Method for Computing Profile-Likelihood-Based Confidence Intervals," Applied Statistics, 37, 87-94.

Welsh, A. H., Cunningham, R. B., Donnelly, C. F., and Lindenmayer, D. B. (1996), "Modelling the Abundance of Rare Species: Statistical Models for Counts With Extra Zeros," Ecological Modelling, 88, 297-308. 